\title{
November 2018 Imaging Case of the Month: Respiratory Failure in a 36-Year-Old Woman
}

\author{
Michael B. Gotway, MD \\ Department of Radiology \\ Mayo Clinic Arizona \\ Scottsdale, AZ USA
}

\begin{abstract}
Clinical History: A 36-year old woman presented with complaints of shortness of breath and worsening dyspnea on exertion. She had a reported history of central nervous system vasculitis of uncertain etiology, treated with azathioprine and prednisone currently, and cyclophosphamide in the past. Her symptoms reportedly responded well to this regimen. Her diagnosis of central nervous system vasculitis was established 6 months earlier when the patient presented with upper extremity paresthesia, headache, left arm weakness, diplopia, and a right eye visual field deficit, evidently with brain imaging showing some pathologic changes, although those records were not available at her presentation. Reportedly she responded well to her immunosuppressive therapy and her steroid and azathioprine doses had been tapered accordingly. Her past medical history was otherwise remarkable for a history of migraine headaches, depression, childhood asthma, hemorrhagic cystitis due to cyclophosphamide (which prompted discounting this drug in favor of azathioprine for the purported central nervous system vasculitis) in the past, and endometriosis.
\end{abstract}

The patient is a former smoker for a total of 5 pack-years, quitting years previously. She is the mother of a 3-year-old child. The patient denied alcohol and drug use. A history of penicillin allergy was elicited. In addition to azathioprine and prednisone, her medications included inhaled budesonide, Bactrim, escitalopram, topiramate, and sumatriptan/naproxen sodium as well as a multivitamin. There was some history of fenfluramine/phentermine ("Fen-Fen") use years earlier.

Her physical examination was largely unremarkable. The patient complained of head pain and was visibly mildly dyspneic, but her lungs were clear and no abnormal heart sounds were detected. Her extremities appeared normal- no ecchymosis, cyanosis, or clubbing was detected. She did have some prior history suggesting the presence of erythema nodosum, now presenting as an erythematous region on the right lower extremity, which underwent biopsy, although changes characteristic of erythema nodosum were not present at her current examination. Reportedly this region had been injured when she bumped the right lower extremity on a chair, and this injury evidently became infected, requiring drainage, yielding cultures positive for Staphylococcus aureus and, about 1 month later, Actinomyces israelii. Her vital signs should normal pulse rate and blood pressure, breathing at 26 breaths / minute. Her room air oxygen saturation was $93 \%$.

Frontal and lateral chest radiography (Figure 1) was performed. 


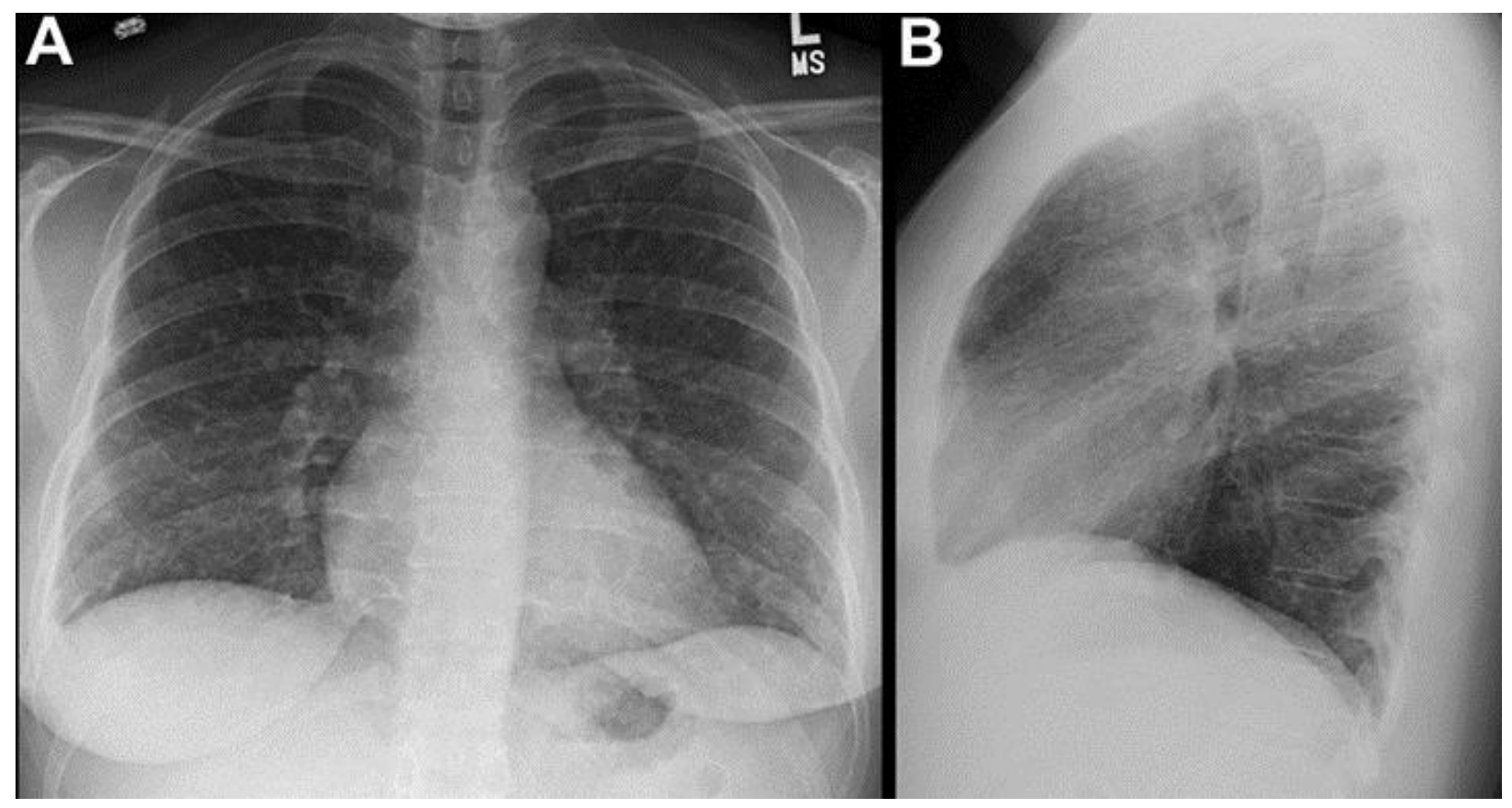

Figure 1. Frontal $(A)$ and lateral $(B)$ chest radiography.

Which of the following represents the most accurate assessment of the chest radiographic findings?

1. Chest radiography shows basilar fibrotic opacities

2. Chest radiography shows bilateral pleural effusions

3. Chest radiography shows cavitary pulmonary lesions

4. Chest radiography shows marked cardiomegaly

5. Chest radiography shows numerous small nodular opacities 


\section{Correct! \\ 5. Chest radiography shows numerous small nodular opacities}

The frontal and lateral chest radiograph shows multiple, small, bilateral mid- and lower lung predominant nodular opacities, possibly with a faint branching configuration, but no pleural effusion or mediastinal or peribronchial lymph node enlargement is present. Lung volumes appear normal and no basal predominant reticulation to suggest fibrotic lung disease is seen. The hear is upper normal in size, but marked cardiomegaly is not present. While nodular opacities are evident in the lungs bilaterally, no cavitary lesions are seen.

The patient's prior medical records were obtained, and it was learned that the biopsy of lower extremity erythema nodosum grew Actinomyces israelii. At this time in another institution she underwent bronchoscopy for complaints of worsening shortness of breath, with negative results for Pneumocystis, but culture results were still pending.

At this point, which of the following represents the most appropriate step in this patient's management?

1. ${ }^{18}$ FDG-PET scan

2. Chest CT

3. Repeat bronchoscopy with transbronchial biopsy

4. Surgical lung biopsy

5. Thoracic MRA 


\section{Correct! \\ 2. Chest CT}

Chest CT is the most appropriate step in the patient's evaluation, given her respiratory complaints and abnormal chest radiographic findings. Bronchoscopy is also a reasonable choice, but she reportedly recently underwent this procedure, so it may be wise to attempt to obtain these results rather than repeat an invasive procedure. Thoracic MRA is primarily used for assessment of the large arteries of the thorax, which could prove useful given the reported history of central nervous system vasculitis, but there are few data to indicate the need for this procedure at this point. Surgical lung biopsy could prove useful given the chest radiographic findings, but these imaging findings are non-specific and are incompletely characterized at this point, and therefore surgical lung biopsy is premature. ${ }^{18} \mathrm{FDG}$-PET scan is clearly useful for the investigation of pulmonary abnormalities, typically in the context of staging suspected or known malignancies or for the assessment of indeterminate lung nodules, but is usually employed after characterization of pulmonary abnormalities with chest CT; therefore, ${ }^{18} \mathrm{FDG}-\mathrm{PET}$ scan is also premature at this juncture.

The patient's bronchoscopy results reportedly showed possible Actinomyces israelii, and apparently, she was started on clindamycin (due to her penicillin allergy) and amphotericin to treat this presumed infection.

A chest CT performed during this hospitalization several months earlier (Figure 2) was located.
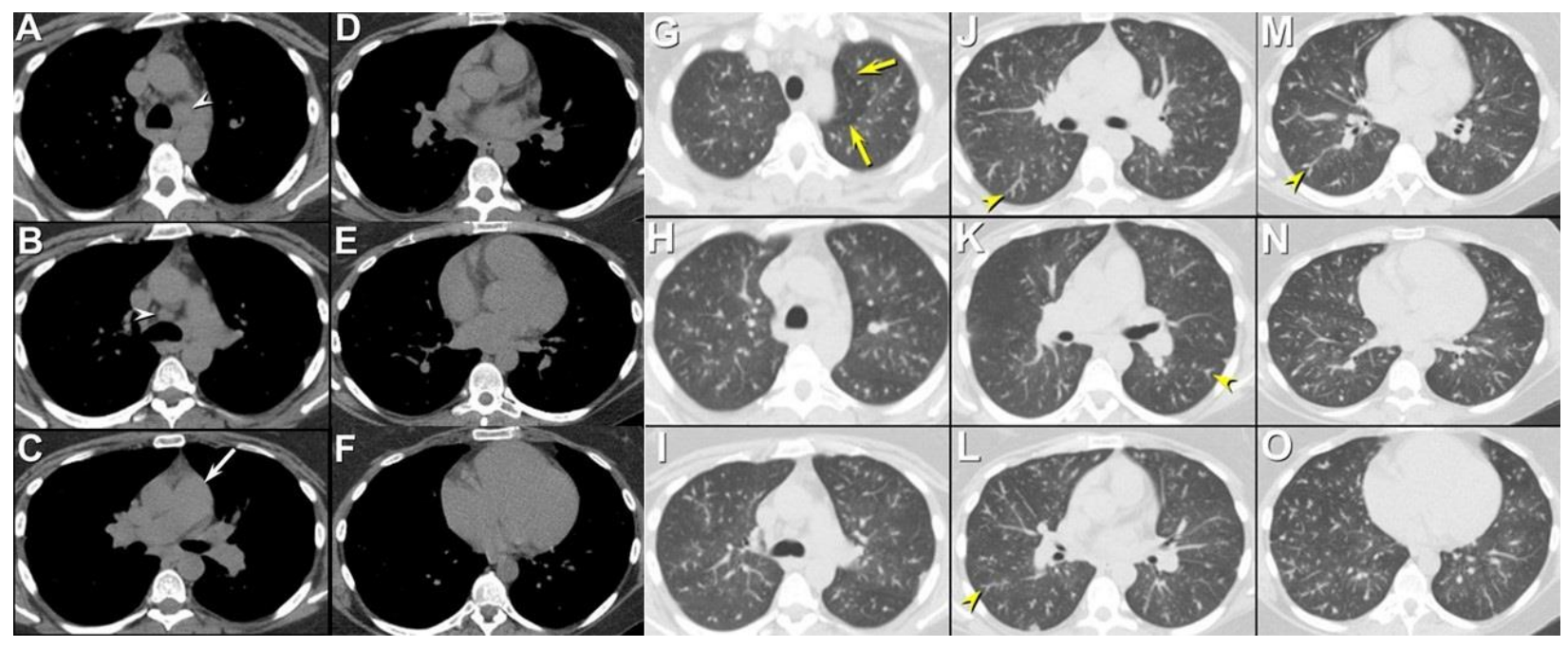

Figure 2. Axial unenhanced thoracic CT displayed in soft tissue windows $(A-F)$ and in lung windows (G-O). 
Which of the following represents the most accurate assessment of the chest CT findings?

1. Chest CT shows multifocal bilateral pleural thickening

2. Chest CT shows multifocal bronchiectasis

3. Chest CT shows numerous small cavitary pulmonary nodules

4. Chest CT shows numerous, small, branching non-cavitary pulmonary opacities

5. Chest CT shows patchy, multifocal areas of ground-glass opacity and consolidation 


\section{Correct!}

\section{Chest CT shows numerous, small, branching non-cavitary pulmonary opacities}

The chest CT shows numerous small nodular opacities, many of which show a somewhat branching configuration, as well as multifocal mosaic perfusion. The small nodular opacities are widely distributed in a random fashion, but no evidence of cavitation is seen. No areas of ground-glass opacity or consolidation is present. The visible airways are not dilated. There are branching opacities, which raises the possibility of airway impaction, but clear evidence of bronchiectasis is lacking. There is no evidence of pleural thickening or effusion.

The patient's shortness of breath was increasing and she was started on oxygen therapy and bilevel positive airway pressure (BiPAP). Laboratory data showed normal renal function, normal liver function, no anemia, and no evidence of an elevated white blood cell count. The report of an echocardiogram performed several months earlier showed normal left ventricular systolic function but right ventricular systolic pressure was estimated to be $70 \mathrm{mmHg}$.

Which of the following represents the most appropriate step in this patient's management?

1. ${ }^{68} \mathrm{Ga}$-Dotatate scan

2. High-resolution chest CT (HRCT)

3. Surgical lung biopsy

4. Thoracic MRI

5. Ventilation - perfusion scintigraphy 


\section{Correct! \\ 5. Ventilation - perfusion scintigraphy}

Among the choices listed, ventilation - perfusion scintigraphy (V/Q scan) is the best choice because the patient's worsening shortness of breath and elevated pulmonary arterial pressure raise the possibility of thromboembolic disease, although this consideration is but one of a number of possibilities for the patient's condition. Surgical lung biopsy is as yet premature, and could engender a higher-than-average risk for complications given the pulmonary hypertension. Thoracic MRI would probably add little to what is known already from the unenhanced thoracic CT, and certainly would not yield additional information regarding the pulmonary abnormalities. ${ }^{68} \mathrm{Ga}$-Dotatate scanning is useful for the assessment of neuroendocrine malignancies, which is not as yet a primary consideration for this patient's condition.

The patient underwent ventilation-perfusion scanning (Figure 3).

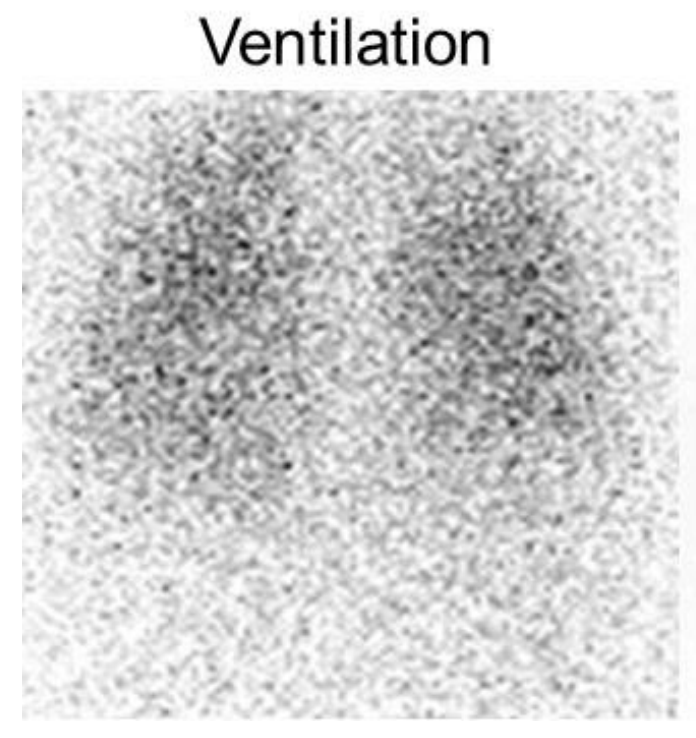

\section{Perfusion}

Figure 3. Ventilation and perfusion scintigraphy (posterior views).

Which of the following represents the most accurate assessment of the ventilationperfusion scan findings?

1. Ventilation - perfusion scintigraphy shows normal findings

2. Ventilation - perfusion scintigraphy shows findings suggesting a low probability of thromboembolic disease

3. Ventilation - perfusion scintigraphy shows findings suggesting an intermediate probability of thromboembolic disease

4. Ventilation - perfusion scintigraphy shows findings suggesting a high probability of thromboembolic disease

5. Ventilation - perfusion scintigraphy shows pulmonary-to-systemic arterial shunting 


\section{Correct! \\ 4. Ventilation - perfusion scintigraphy shows findings suggesting a high probability of thromboembolic disease}

The ventilation-perfusion scan shows a number of small, peripheral perfusion defects without a corresponding ventilation abnormality, suggestive of a pulmonary vascular obstructive process, which is typical of thromboembolic disease. The ventilation and perfusion "mis-matching" is consistent with a high probability for thromboembolic disease. No evidence of tracer uptake over the liver or cranial kidneys is seen to suggestive right-to-left tracer shunting.

At this point, which of the following represents the most likely diagnosis for this patient's condition?

1. Collagen vascular disease

2. Septic embolization due to actinomycosis affecting the lower extremity

3. Thromboembolic disease

4. Vasculitis

5. No particular diagnosis can be offered 


\section{Correct! \\ 5. No particular diagnosis can be offered}

Any of the possibilities mentioned are possible explanations for the patient's condition, but data sufficient to establish a specific diagnosis is lacking. Obtaining Actinomyces israelii from both the lower extremity skin biopsy and from bronchoscopy raises the possibility of a systemic infection, particularly in the context of immunosuppression, but the chest CT findings are certainly atypical in this regard and the bronchoscopic findings could merely reflect contamination. Furthermore, the pulmonary hypertension remains unexplained by actinomycosis. The ventilation-perfusion scintigraphy results suggest thromboembolic disease, which could also explain the pulmonary hypertension, but the lung findings at chest CT are inconsistent with thromboembolic disease. Vasculitis and a collagen vascular disease both could explain the pulmonary hypertension as well as the patient's presentation, but the presence of Actinomyces israelii and the pulmonary findings at chest CT are inadequately accounted for by these disorders. Ultimately more investigation is required.

Given the discordant data, the patient underwent CT pulmonary angiography (Figure 4) to assess for acute or chronic thromboembolic disease.

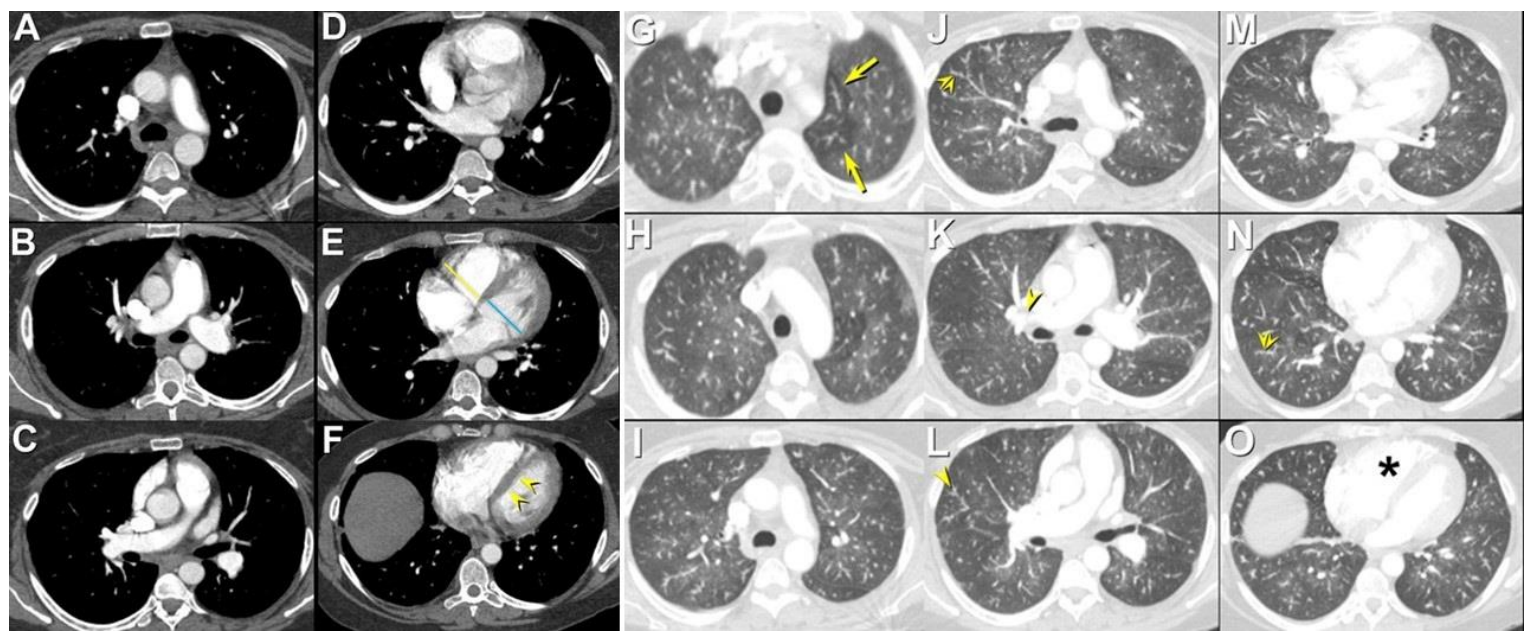

Figure 4. Axial enhanced CT in soft tissue windows $(A-F)$ and lung windows $(G-O)$.

Which of the following represents the most accurate assessment of the chest CT findings?

1. CT pulmonary angiography shows acute pulmonary emboli

2. CT pulmonary angiography shows chronic pulmonary emboli

3. CT pulmonary angiography shows progression of the abnormalities seen at the previous unenhanced chest CT (Figure 2)

4. CT pulmonary angiography shows regression of the abnormalities seen at the previous unenhanced chest CT (Figure 2)

5. Pulmonary angiography shows no change in the pulmonary parenchymal findings seen at the unenhanced chest CT (Figure 2) 


\section{Correct! \\ 5. Pulmonary angiography shows no change in the pulmonary parenchymal findings seen at the unenhanced chest CT (Figure 2)}

The CT pulmonary angiogram shows no evidence of acute or chronic thromboembolic disease, although features of increased right heart pressures, evidenced by straightening of the interventricular septum and enlargement of both the main pulmonary artery and right ventricle, are present. The randomly distributed, branching nodular opacities noted throughout the lung parenchyma remain unchanged.

The patient underwent lower extremity venous ultrasound examination, which was normal. Review of the outside facility bronchoscopy with transbronchial biopsy showed a few non-specific granulomas, and only one washing showed Actinomyces israelii and it was felt therefore that this organism may simply be a contaminant. The patient's erythrocyte sedimentation rate and C-reactive protein levels were elevated at $50 \mathrm{~mm} / \mathrm{h}$ (normal, $<10 \mathrm{~mm} / \mathrm{h}$ ), and $23 \mathrm{mg} / \mathrm{L}$ (normal, $<5 \mathrm{mg} / \mathrm{L}$ ), respectively. The patient underwent repeat brain MRI (Figure 5), which showed subcortical abnormalities in both the supratentroial and infratentorial compartments suggesting embolic infarction.

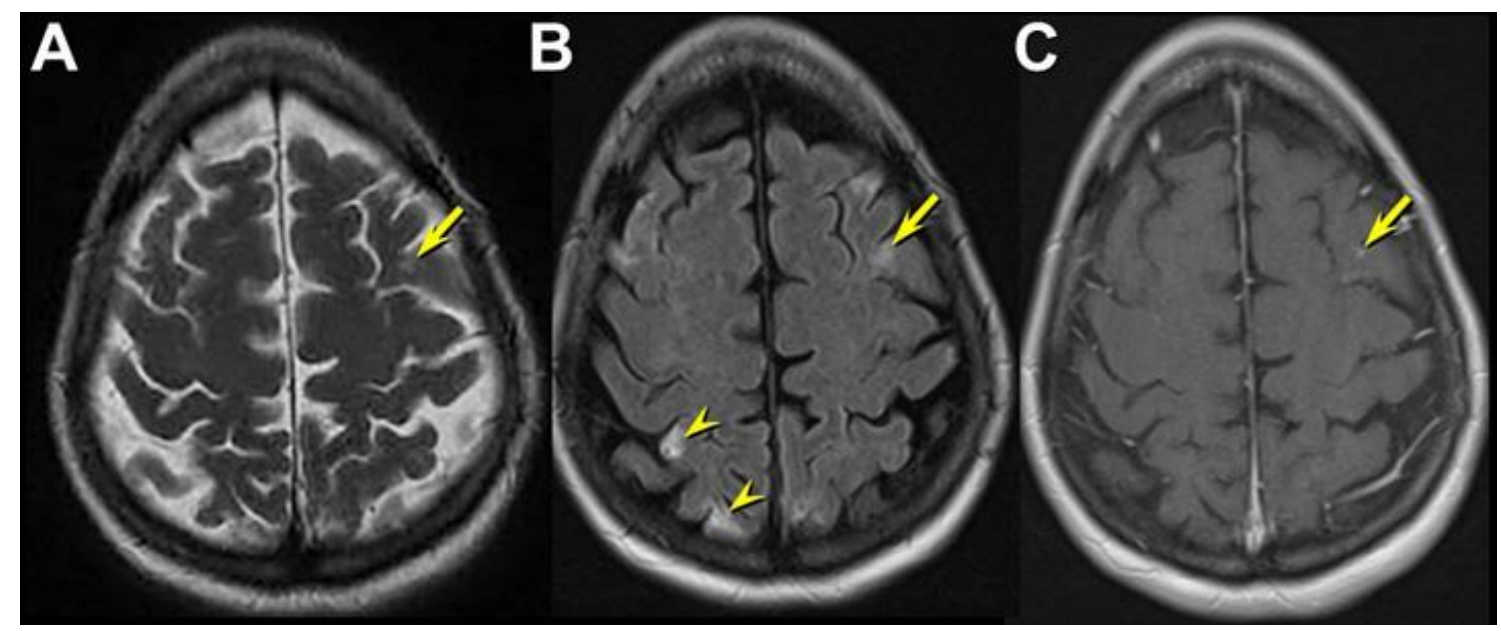

Figure 5. Axial T2-weighted (A), fluid sensitive (aka "FLAIR," B), and contras-tenhanced T1-weighted brain MR just cranial to the lateral ventricular system, shows subcortical areas of T2-hyperintensity (arrow, A) that are also hyperintense on fluidsensitive imaging (arrowheads and arrow, B), with subtle areas of enhancement (arrow, C).

At this point, which of the following represents the most appropriate step in this patient's management?

1. MRA of the brain and head and neck vessels

2. Neurology consultation

3. Repeat echocardiogram

4. Rheumatology consultation

5. All of the above 


\section{Correct! \\ 5. All of the above}

Given the complexity of the patient's condition, all of the above actions are appropriate choices at this point in the patient's presentation.

Infectious disease, Neurology, and Rheumatology were consulted, with recommendations including brain and head and neck MRA (given the history of central nervous system vasculitis and recent brain MRI results), repeating the echocardiogram, continuing the corticosteroid therapy but discounting antibiotic therapy to increase the likelihood of finding a causative organism, obtaining blood and sputum cultures, testing for tuberculosis, biopsying the lower extremity rash (now somewhat pruritic and nodular), sampling of the cerebrospinal fluid, and obtaining some additional laboratory data, particularly targeting rheumatological diseases and vasculitis.

Most of the patient's rheumatological studies, including the patient's anti-phospholipid antibody levels and cytoplasmic anti-neutrophil cytoplasmic antibody studies were negative, although the anti-nuclear antibody titer was elevated at 1:160, with a speckled pattern. Repeat transthoracic echocardiography confirmed pulmonary hypertension with preserved left ventricular systolic function, but no thrombus was seen within the left atrium or left atrial appendage. Brain and head and neck MRA showed no evidence of vascular abnormalities to suggest plaque or vasculitis; no embolic source was seen. Comparison of the recent brain MRI to a study performed 6 months earlier at an outside institution, however, revealed that some of the changes noted currently were new compared with that previous examination. Cerebrospinal fluid analysis from the lumbar puncture was showed no abnormalities; no evidence of an elevated white blood cell count or pleocytosis was noted in the cerebrospinal fluid. Blood cultures showed no growth at 3 days, testing for tuberculosis was negative, and testing for fungal antigens was also negative.

At this point, which of the following represents the most appropriate step in this patient's management?

1. Cerebral angiography

2. Brain biopsy

3. Right heart catheterization

4. 1 and 3

5. All of the above 


\section{Correct}

\section{1 and 3}

Formally assessing the patient for pulmonary hypertension with right heart catheterization is appropriate given the remote history of fenfluramine/phentermine ("Fen-Fen") use as well as two echocardiograms, and a chest CT, showing features suggesting pulmonary hypertension. Cerebral angiography is appropriate to assess for vasculitis and embolic sources, particularly given the brain MRI showing embolic phenomenon. While the recent brain and head and neck MRA showed no evidence of either vasculitis or embolic sources, catheter cerebral angiography may be more sensitive for the detection of such abnormalities. Brain biopsy is not indicated as the patient reportedly responded well to immunosuppressive therapy for presumed central nervous system vasculitis and the risk of performing a brain biopsy may not be outweighed by any information obtained by the procedure, at least at this patient with the patient's presentation. Furthermore, the appearance of the brain MRI is more suggestive of an embolic phenomenon than a cerebral vasculitis, and the cerebral angiogram results may further inform the need for brain biopsy and should be performed before this invasive procedure is undertaken in this circumstance.

Cerebral angiography was performed and showed no abnormalities. Neurosurgery was consulted but declined brain biopsy, and neurology concurred with this assessment. Cardiology was consulted and right heart catheterization was performed, which showed a low cardiac index of $1.5 \mathrm{~L} / \mathrm{min} / \mathrm{m}^{2}$ and a mean pulmonary arterial pressure of 62 $\mathrm{mmHg}$, the latter unaffected by nitric oxide infusion.

The pulmonary capillary wedge pressure was normal. At this point, which of the following represents the most appropriate step in this patient's management?

1. Begin amiodarone therapy

2. Begin calcium channel blocker therapy

3. Begin intravenous epoporostenol therapy

4. Begin milrinone therapy

5. Begin $\beta$-blocker therapy 


\section{Correct! \\ 3. Begin intravenous epoporostenol therapy}

The patient's right heart catheterization results suggest group I pulmonary arterial hypertension, for which prostacyclin therapy is appropriate. Anti-dysrhythmic agents are not indicated, nor is therapy directed at decreasing heart rate and left ventricular afterload or for augmenting left ventricular contractility.

The biopsy of the lower extremity rash showed fat necrosis, thought to be the result of trauma, and some eosinophilia, but no organisms were identified and no features diagnostic of a particular condition were seen. Tadalafil was added to the patient's pulmonary hypertension regimen. The patient began to improve, with no fever development and decreasing oxygen requirements, eventually oxygenating well on room air. Repeat transthoracic echocardiogram showed decrease in the right ventricular systolic pressure from $88 \mathrm{mmHg}$ to $66 \mathrm{mmHg}$. Subsequently, the patient underwent transesophageal echocardiography which showed normal left ventricular systolic function. Her right ventricular systolic pressure was $62 \mathrm{mmHg}$. No masses, thrombus, or vegetation on the valves were noted. A patent foramen ovale was noted.

At this point, which of the following represents the most appropriate step in this patient's management?

1. Brain biopsy

2. Myocardial biopsy

3. Percutaneous transthoracic needle biopsy

4. Repeat bronchoscopy with bronchoalveolar lavage and transbronchial biopsy

5. Surgical lung biopsy 


\section{Correct! \\ 5. Surgical lung biopsy}

Given the pulmonary parenchymal findings, a surgical lung biopsy may be the single best procedure to obtain a unifying diagnosis for this patient. Brain biopsy is quite invasive, and, as noted previously, may not yield the desired information, particularly given that treatment, which could alter biopsy results, has been instituted for some time. Additionally, the likelihood of central nervous system vasculitis is now low, in light of the lumbar puncture and cerebral angiogram results; rather, an extracranial embolic source for the brain parenchymal changes seems more likely. Bronchoscopy with bronchoalveolar lavage and transbronchial biopsy is a reasonable thought, but has been performed recently without a definitive diagnosis, and therefore the yield of repeating this procedure is low, and the review of the material performed at the bronchoscopy at the outside facility just prior to the patient's admission is still pending. Percutaneous transthoracic needle biopsy of the lung is typically utilized for focal lesions, not for the diffuse abnormalities affecting this patient's lung parenchyma. Myocardial biopsy may be used in patients with heart transplant to assess for rejection or for patients with myocardial dysfunction thought to the related to myocarditis or nonischemic cardiomyopathy, but this patient's left ventricular mechanics are normal, and biopsying the right heart would not provide data that could establish the etiology of her pulmonary hypertension. A surgical lung biopsy, despite associated risks given the pulmonary hypertension, is probably the best procedure for establishing the etiology of the pulmonary hypertension and abnormalities seen at chest CT and perhaps even the brain MRI findings, the latter particularly in light of the patent foramen ovale. Note that cryobiopsy could play a role here as a less invasive means for obtaining lung tissue for diagnosis compared with surgical biopsy, but this choice was not listed above.

The patient was scheduled to undergo video-assisted thoracoscopic lung biopsy, but lung biopsy material obtained from her outside facility bronchoscopy with transbronchial biopsy showed perivascular refractile crystalline material consistent with microcrystalline cellulose, associated with granulomas suggesting a foreign body reaction. This discovery prompted a pointed discussion with the patient, who then admitted to crushing hydrocodone tablets and injecting them intravenously; she had previously denied substance abuse, even when directly asked pointed questions regarding the injection of illicit drugs. The open lung biopsy procedure was deferred, as the patient's pulmonary hypertension and central nervous system abnormalities are readily explained by intravenous injection of a foreign substance causing pulmonary arteriolar obstruction and pulmonary hypertension, with the patient foramen ovale allowing systemic embolization of the injected material, resulting in embolic cerebral infarction. Psychiatry was consulted to assist in management of the patient's substance abuse.

Diagnosis: Pulmonary hypertension and cerebral infraction resulting from intravascular embolization of hydrocodone in an injection drug user with a patent foramen ovale 


\section{References}

1. Ward S, Heyneman LE, Reittner P, Kazerooni EA, Godwin JD, Müller NL. Talcosis associated with IV abuse of oral medications: CT findings. AJR Am J Roentgenol. 2000;174(3):789-93. [CrossRef] [PubMed]

2. Bendeck SE, Leung AN, Berry GJ, Daniel D, Ruoss SJ. Cellulose granulomatosis presenting as centrilobular nodules: CT and histologic findings. AJR Am J

Roentgenol. 2001;177(5):1151-3. [CrossRef] [PubMed]

3. Gotway MB, Marder SR, Hanks DK, Leung JW, Dawn SK, Gean AD, Reddy GP, Araoz PA, Webb WR. Thoracic complications of illicit drug use: an organ system approach. Radiographics. 2002; 22 Spec No:S119-135. [CrossRef] [PubMed] 